\title{
A psychoeducational program increased knowledge and decreased sexual risk behaviors in young adults with genital herpes
}

\author{
JM Swanson, \\ SL Dibble, \\ LChapman \\ J Adv Nurs \\ 1999;29:840-851. \\ Correspondence to: \\ Dr J M Swanson \\ Department of Nursing \\ Samuel Merritt College \\ 370 Hawthorne Ave \\ Oakland, CA 94609 \\ Funding: National \\ Institute of Nursing \\ Research; Research \\ Center for Symptom \\ Management, University \\ of California \\ This paper was originally \\ published in \\ Evidence-Based Nursing \\ 2000;3:15.
}

\section{QUESTION}

In young adults with genital herpes, does a group psychoeducational intervention led by nurses decrease sexual health risk and improve psychosocial adaptation?

\section{DESIGN AND SETTING}

Randomized (allocation concealed),* unblended, controlled trial of 6 months' duration in a metropolitan area in California.

\section{PARTICIPANTS}

A total of 252 adults (mean age: 27 years; $77 \%$ women; $80 \%$ white; $70 \%$ single; $84 \%$ employed; $10 \%$ lesbian or gay; mean education: 15 years; and mean annual income: $\$ 22,000)$ with confirmed symptomatic genital herpes diagnosed within the previous 5 years were included in the study. Adults with histories of inpatient treatment for psychiatric conditions or alcohol or drug misuse were excluded. Follow-up was $87 \%$.

\section{INTERVENTION}

One hundred thirty-one adults were allocated to group psychoeducational sessions (3 sessions, 90 minutes each) led by a nurse. Problem solving, skills building, and peer and professional support were emphasized. Topics included information and feelings about herpes, how to disclose the diagnosis to others, preventing the transmission of the disease, safe sexual behavior, and substance use. Resource materials were provided. One hundred twentyone adults were offered the same intervention after the 6-month study was complete (control group).

\section{MAIN OUTCOME MEASURES}

Sexual health risks measured using the Herpes Knowledge Scale to assess knowledge, the Sexual Health Risks questionnaire to measure sexual behavior (condom and spermicide use, number of sexual partners, and disclosure of herpes status), the Protection from Sexually Transmitted Disease questionnaire to measure self-efficacy about the prevention or transmission of genital herpes and other sexually transmitted diseases, and psychosocial adaptation measured using the Profile of Mood States checklist and Beck Depression Inventory.

*Information provided by JM Swanson

\section{MAIN RESULTS AND CONCLUSIONS}

All analyses were adjusted for baseline scores. By 6 months, adults in the psychoeducational intervention group had higher knowledge scores (mean: $95 \%$ vs $91 \%$ correct; $\mathrm{P}<0.001$, used condoms more often $(87 \%$ vs $74 \%$ of the time; $\mathrm{P}<0.001)$, used spermicide more often $(41 \%$ vs $20 \%$ of the time; $\mathrm{P}<0.001$ ), and planned to tell potential sexual partners about herpes more often $(\mathrm{P}=0.02)$. Similar results were shown at 3 months. The groups did not differ for number of sexual partners in the previous 3 months; number, length, or severity of outbreaks in the previous 3 months; mood states (anxiety, depression, anger, fatigue, vigor, or confusion); total mood disturbance; depression; or feelings of self-efficacy to prevent the transmission of sexually transmitted diseases.

A psychoeducational intervention led by a nurse for young adults with genital herpes increased knowledge of the disease and decreased sexual risk behaviors, but did not improve psychosocial adaptation.

\section{Commentary}

Colleen Van Berkel, STD/HIV Program, Hamilton-Wentworth Regional Social and Public Health Services Division, Hamilton, Ontario, Canada

Genital herpes is a chronic viral sexually transmitted disease. Stressors associated with the disease include the fear of telling new sexual partners $^{1}$ and depression. ${ }^{2}$

Psychoeducational interventions are used to increase coping skills, acceptance of the illness, and cooperation with treatment and rehabilitation. ${ }^{3}$ This type of intervention has been used successfully to address sexual issues such as AIDS and sexually transmitted diseases. ${ }^{4}$

The generalizability of the study to other populations is limited because the sample included English-speaking literate participants, of whom $77 \%$ were women and $80 \%$ were white. Six months of follow-up was short, and no cost-effectiveness analysis of the psychoeducational intervention was provided. Although the intervention was successful in decreasing sexual health risks, it did not have an effect on any of the measures of psychosocial adaptation. This was probably because the participants in this study were already in the normal range for these measures.

1 Keller M, Jadack R, Mims R. Perceived stressors and coping response in persons with recurrent genital herpes. Res Nurs Health 1991;14:421-430.

2 Drob S, Bernard $\mathrm{H}$. Two models of brief group psychotherapy for herpes sufferers. Croup 1986;9:133-144.

3 Goldman CR. Toward a definition of psychoeducation. Hosp Community Psychiatry 1988;39:666-668.

4 Steiner JL, Lussier RG, Maust GC, et al. Psychoeducation about sexual issues in an acute treatment setting. Hosp Community Psychiatry 1994;45:380-381. 